\title{
Concierto y desconcierto en la organización del conocimiento actual y su intersección con el mundo de la información
}

\section{Emilia Currás}

Académica. IIS Fellow

Universidad Autónoma de Madrid

E1 "misterio" es fruto de la ignorancia. Todavía existen "misterios" porque los humanos aún somos muy ignorantes. Por medio del conocimiento, poco a poco, iremos descubriendo los velos de la ignorancia ( E. C.)

\subsection{Resumen}

Se hace un estudio del concepto de organización del conocimiento, poniendo de manifiesto su evolución desde concepciones puramente metafísicas, especulativas, hacia otras puramente físicas y pragmáticas-informáticas. Se relacionan la información, el conocimiento y las tecnologías de la información que actúan en ese cambio de paradigma de la organización del conocimiento desde la perspectiva de las Ciencias de la Documentación y su implantación como asignatura en los estudios universitarios. (Autor)

Palabras clave: Organización de Conocimiento, Ciencias de la Documentación, Información Científica.

\subsection{Abstract}

The concept of knowledge organisation is explored from the point of view of Information Science, showing its evolution from pure metaphysic and especulative thinking toward physical, informational and pragmatically-oriented positions. The relation among information, knowledge and information technologies is considered to be a key aspect of this paradigm shift from the perspective of Information Science. Finally, the possibity of teaching a specific subject on Knowledge Organisation in Spanish universities is considered. (Author)

Keywords: Knowledge Organisation, Library and Information Science. 


\section{Introducción}

Escribir sobre el tema de la organización del conocimiento, en estos momentos históricos de transición hacia nuevas formas de pensamiento, resulta sumamente atractivo y también sumamente arriesgado. Por un lado, los conceptos se amplían enfocando mayores niveles de abstracción, por otro lado, los continuos inventos y descubrimientos suscitan la aparición de nuevas disciplinas, que no obstante, no quedan aisladas en el concierto de saberes. Se integran, se entrelazan, llegando a confundirse sus límites. No resulta fácil fijar fronteras y menos aún formular definiciones. Los mismos especialistas no aciertan a concretar paradigmas, ni metáforas sobre las que fundamentar sus postulados, opiniones e investigaciones.

Algunas veces se tiene la impresión de que los intelectuales actuales, científicos o humanistas, no terminan de abarcar el conjunto de sus propias especializaciones, ni llegan a penetrar en las intersecciones de sus temas de trabajo con otras ramas del saber. Podría parecer que se aprecia una cierta superficialidad en las opiniones expuestas. Quizá también sean avances hacia nuevas ideologías, nuevos paradigmas; por nuevos, aún no bien concretados.

Uno de los temas más convulsionados en los tiempos presentes y que ha dado motivo a las reflexiones precedentes es el relativo a la "organización de conocimiento". Precisamente por ello, es arriesgado escribir sobre él. Por otra parte, ante tal diversidad de opiniones, conceptos postulados... no se puede uno sustraer, a llevar a cabo, al menos, una relación aséptica de algunas de aquellas. Se trata de intentar traer algo de claridad a un campo del saber en plena evolución y por ende en estado de desequilibrio.

\section{Desarrollo del tema}

Cuando se tiene la intención de tratar un tema, suele ser recomendable concretar las bases sobre las que se van a asentar los razonamientos desarrollados. Así pues, en este caso se debería empezar definiendo qué se entiende por "organización del conocimiento”. Pero ¿cómo hacerlo sin fijarse en el propio "conocimiento" origen y causa de su misma organización?

El conocimiento está íntimamente relacionado con la información, y ésta con las ciencias de la documentación (o documentarias), con la informática, las nuevas tecnologías de la información... y otras varias materias. De ahí, que se hable primero de ellas para sentar las bases sobre las que sustentar nuestra "organización del conocimiento".

El tema se trata en tono dubitativo, en un cierto modo fluctuante, utilizando la forma condicional, con la intención de no desear adoptar premisas, ni opiniones rotundas. Se deja lugar a la controversia y la crítica.

Scire. 1 : 1 (en.-jun. 1995). 
Se debe aclarar que se entiende la ciencia como unificación de saberes, donde quedan implícitas, tanto las ciencias naturales, como las humanistas. Cultura supone un nivel mayor de abstracción, concebida como ciencia acumulada y asimilada por un pueblo. Por tanto, cuando se mencionan los "investigadores y pensadores científicos y humanistas" se hace para mejor entendimiento de los posibles lectores. La idea de ciencia unidad todavía no ha sido ni suficientemente difundida, ni aceptada.

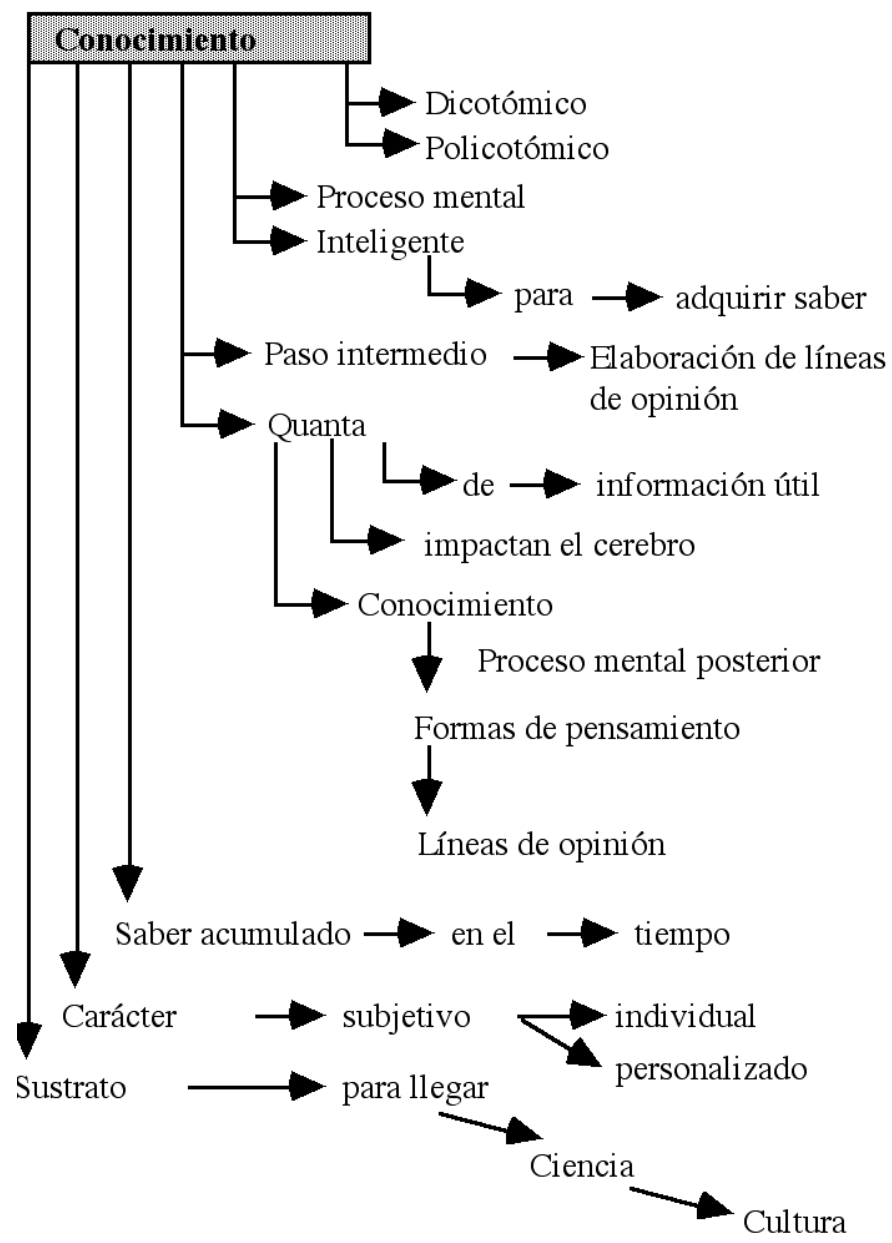

Figura 1

Scire. $1: 1$ (en.-jun. 1995). 


\section{Concepto de conocimiento}

Realizar un estudio exhaustivo del concepto de conocimiento, podría ser tarea ardua, ya que intelectuales y pensadores, tanto científicos como humanistas, de todos los tiempos, han aventurado muy diversas opiniones y definiciones, cada una desde un punto de vista diferente. La bibliografía existente es muy abundante. Aquí se va a hacer referencia a aquellas definiciones y opiniones que sirven al propósito de este escrito.

El vocablo "conocimiento" pertenece al grupo de los dicotómicos, o quizá también de los policotómicos, si consideramos su cuasi-sinonimia con cultura, saber, ciencia... y otros términos semejantes.

Conocimiento se puede entender como proceso mental, inteligente, para adquirir saber, donde igualmente supondría un paso intermedio en la elaboración de líneas de opinión. Se situaría entre los quanta de información útil que impactan el cerebro para producir conocimiento y su proceso mental posterior, que origina las formas de pensamiento, terminando en las líneas de opinión (Fig. 1).

Por otra parte, se entiende como conocimiento el saber acumulado en el tiempo. Aquí es donde se podría establecer la comparación, o cuasi-sinonimia, con ciencia o con cultura. A mi modo de ver, las diferencias estriban en el carácter subjetivo del conocimiento. Este como tal, es individual y personalizado, depende en cada caso, del "ente" conociente (que realiza el acto de conocer) o continente del conocimiento. La ciencia y la cultura son universales, del dominio público, aunque para llegar a ellas sea necesario el esfuerzo mental de investigadores, aislados o reunidos en grupos. El conocimiento supone un sustrato para llegar a la ciencia y la cultura (Fig. 1).

Los filósofos orientales enfatizan ese carácter subjetivo del conocimiento, así Pandey S. K.Sharma dice que el estudio del conocimiento pertenece al mundo de la especulación, porque no se conocen los mecanismos internos por los que se llega a él. M. A. Gopinath constata que el conocimiento tiene la habilidad (¿facultad?) de consolidar el valioso resultado del pensamiento humano. Otras ideas relacionan el conocimiento con experiencias intelectuales, donde juega un papel muy importante la capacidad de percepción del sujeto conociente, el que realiza el acto de conocer.

Los filósofos occidentales contemplan tanto el aspecto interno humano, como los agentes externos. Se adquiere conocimiento por las sensaciones, o percepciones, que se reciben del exterior y que son interpretadas por cada individuo, según su propia idiosincrasia, añadiendo un componente de racionalidad. Platón en su diálogo con Threatatus ya indica que la creencia se convierte en conocimiento cuando actúa la razón.

A lo largo del Medioevo se empieza a notar una transición desde un proceso

Scire. 1 : 1 (en.-jun. 1995). 
lógico hacia otro en el que se añade la experiencia. Alberto Magno, en el siglo XIII, basa el conocimiento en la experimentación práctica y Ruggero Bacone confronta el conocimiento por argumentación con el de experimentación.

Durante el Renacimiento y épocas posteriores sigue prevaleciendo esa misma idea. Kant en el siglo XVIII comenta que se debe racionalizar la experiencia para adquirir conocimiento cierto.

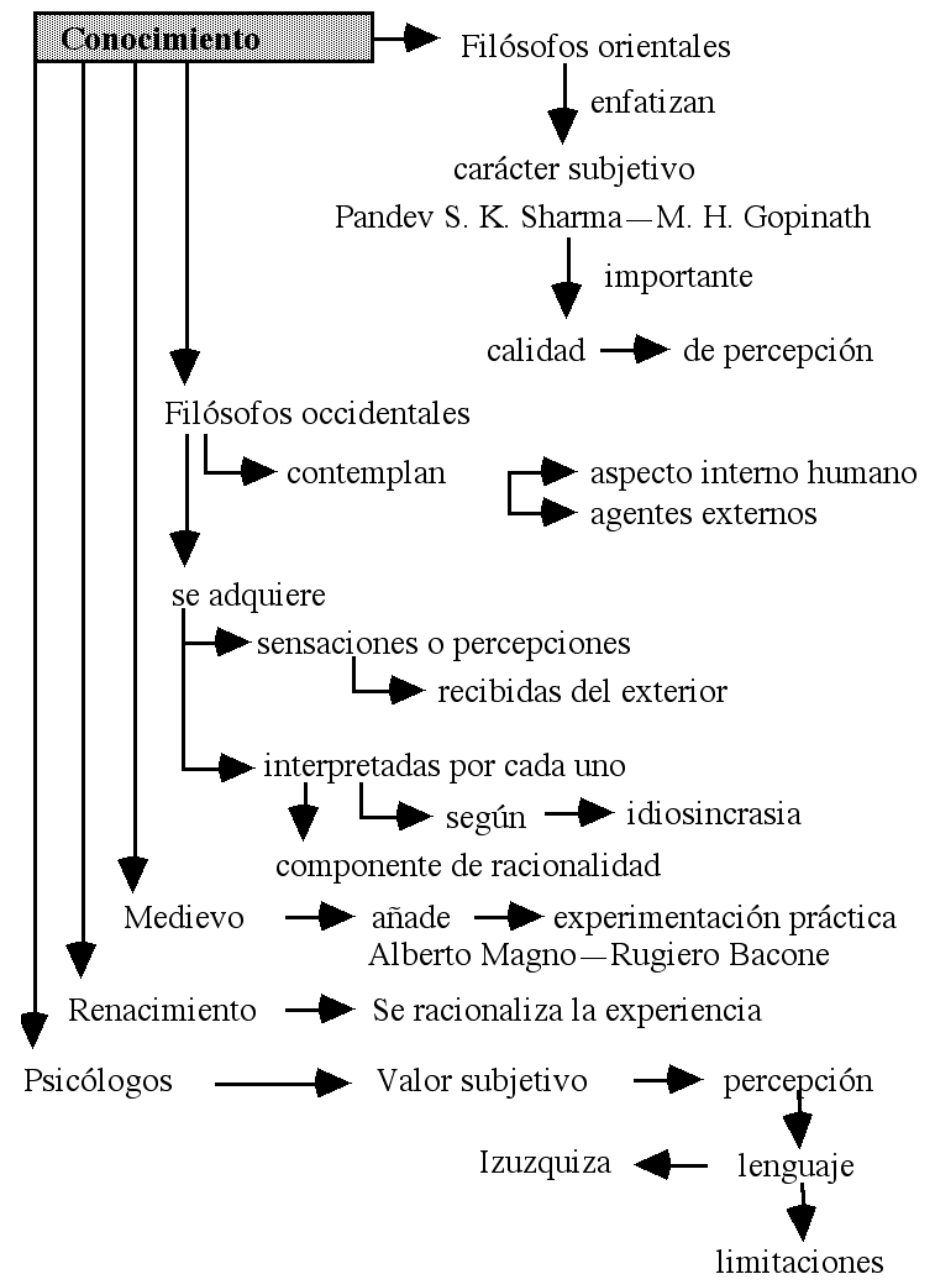

Figura 2

Scire. $1: 1$ (en.-jun. 1995). 
Los psicólogos de estos últimos siglos, con sus estudios sobre el comportamiento humano, hacen hincapié en el valor subjetivo del conocimiento, concentrando sus investigaciones en los mecanismos y motivaciones de la percepción de forma individual y personalizada. El conocimiento se relaciona con experiencias conscientes que siguen inmediatamente a un estímulo de uno o más órganos sensoriales.

Se llega a enlazar percepción con lenguaje, como la genuina forma de expresión, que asimismo sirve para establecer la comunicación con sus semejantes; pero que también puede suponer una limitación a las facultades del hombre. Así Izuzquiza nos dice que el lenguaje establece límites a nuestros pensamientos; E. Coseriu supone que el lenguaje clasifica la realidad limitándola a los intereses y actividades individuales; Petrovsky habla de la consolidación del conocimiento por las palabras, que son esenciales para la actividad del intelecto. David Ausubel se mueve en esa misma línea. De hecho, se ha constatado que los seres humanos elaboramos más ideas que palabras para expresarlas.

La relación entre conocimiento y lenguaje es sumamente sugerente e importante para las ciencias de la documentación, toda vez que se establece una concordancia, sistema, entre aquellos dos conceptos y el de documento, donde se plasma, se conserva para su posterior uso, el conocimiento por medio del lenguaje (Fig 2).

\section{Nuevo concepto de conocimiento}

El concepto de conocimiento cambia cuando empiezan a utilizarse los ordenadores aplicados a los procesos informativos-documentarios. También influye poderosamente la gran difusión del idioma inglés y el uso del vocablo "knowledge", que en sentido estrictamente etimológico significa "conocimiento acumulado" y que en los países anglosajones y aquellos de su área de influencia cultural, emplean también como "ciencia acumulada". Es decir, desaparece el carácter subjetivo, personal, del conocimiento. De ahí asimismo, que se hable de "base de conocimiento" cuando en realidad se trata de una base, o almacén, de datos acumulados en una memoria de ordenador. Padmini Srinivasan riza el rizo cuando habla de "indexing knowledge" = conocimiento de indización.

Cuando Pylyshyn nos anuncia, que el conocimiento debe ser considerado como sinónimo de computación, no anda falto de razón, si se tienen en cuenta los estudios sobre las actividades y funcionamiento del cerebro que se llevan realizando desde hace ya unos cuantos años. Entre ellos cabría mencionar, por originales y recientes, los llevados a cabo por Pedro C. Marijuán y John Westley. Estos autores centran sus estudios en la evolución y actividad de las células del cerebro. Sintetizando, consideran que la célula puede compararse a un sistema

Scire. 1 : 1 (en.-jun. 1995). 
informático. Los RNA pasados a DNA suponen ser la "base de conocimiento", que contienen la información estática almacenada. Los ácidos nucleícos componen el software y las proteínas el hardware. De entre éstas, las enzimas, proteínas no estructuradas, actúan a modo de circuitos electrónicos, a la vez que comportan las reglas de inferencia. Otros grupos de proteínas hacen las funciones de vehículos, de transmisores, del conocimiento elaborado.

Según esto, se supone que la información llega a la célula, actúa como un

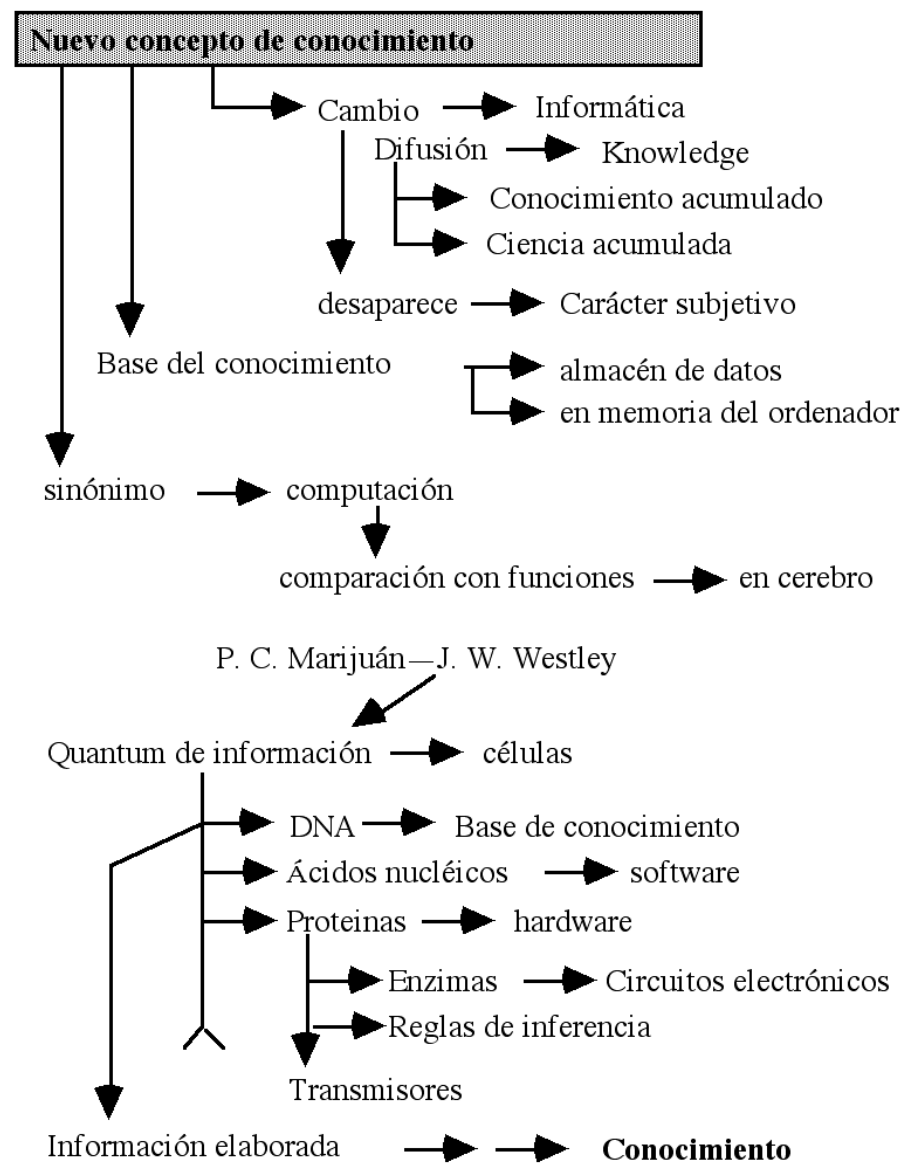

Figura 3

Scire. $1: 1$ (en.-jun. 1995). 
quantum de energía y la activa, de acuerdo con las teorías de Szent Gyòrgyi, entre otros. Entonces se pone en marcha el proceso antes indicado y se obtiene una nueva información elaborada, es decir, conocimiento.

He aquí las nuevas tendencias en las líneas de pensamiento, donde todo gira alrededor de la informática y la cibernética (Fig. 3).

\section{Intersección con la información}

Al hilo de esa manera de interpretar la asimilación de la información por el cerebro y de relacionar aquélla con el conocimiento, parece oportuno mencionar algunas de las ideas de autores recientes, que siguen esas mismas líneas.

De la ya abundante bibliografía merecen destacarse las opiniones de Javier García Marco, quién afirma que el conocimiento es información integrada y personalizada, disponible para la acción; la información es conocimiento potencial en forma directamente proporcional a su accesibilidad. Esto supone establecer un circuito conocimiento-información de movimiento lineal; pero discontinuo en el tiempo y en el espacio, donde ambos componentes forman un sistema, actuando la información como vector de entrada y de salida, al mismo tiempo que elemento de retroalimentación. Esta visión sistémica es muy novedosa e interesante. Por su parte, Padmini Srinivasan nos dice que el conocimiento se incluye en un sistema de recuperación de información, pues permite representar y operar con los objetos básicos de los textos; es decir, los datos (nota de la autora). Otra opinión relacionada con la anterior se debe a Newel. Para él conocimiento es lo que está comprendido en una representación simbólica de información (Fig 4).

Ranganathan, cuyo primer centenario de su nacimiento se ha celebrado en 1992 con gran solemnidad en la India, tiene una visión más teórico-psicológica, ya que postula que conocimiento es información depositada en la memoria como resultado de la asociación de percepciones. En varios escritos yo misma he relacionado ambos conceptos, tomándolos en sentido contrario de influencia. La información es causa primera para producir conocimiento, cuando llega al cerebro e impacta las neuronas. Entonces comienzan a tener lugar, de forma sucesiva, o quizá simultánea al mismo tiempo, ya que nuestra medida del tiempo se ajusta sólo a nuestra dimensión espacio-temporal mesocósmica; procesos de percepción, aprehensión, análisis, clasificación, archivo en memoria, evaluación... que constituyen el conocimiento personal, subjetivo y condicionado por el sustrato individual y cultural de cada individuo. En una elaboración mental posterior, más compleja, el conocimiento pasa a constituir las ideas, líneas de pensamiento. Estas son las que vuelven a convertirse en información útil, cuando surge la ocasión. Aquí también, se forma un sistema, aunque bastante más complejo, ya que se sabe tener en cuenta un mayor número de elementos, parámetros, varia-

Scire. 1 : 1 (en.-jun. 1995). 


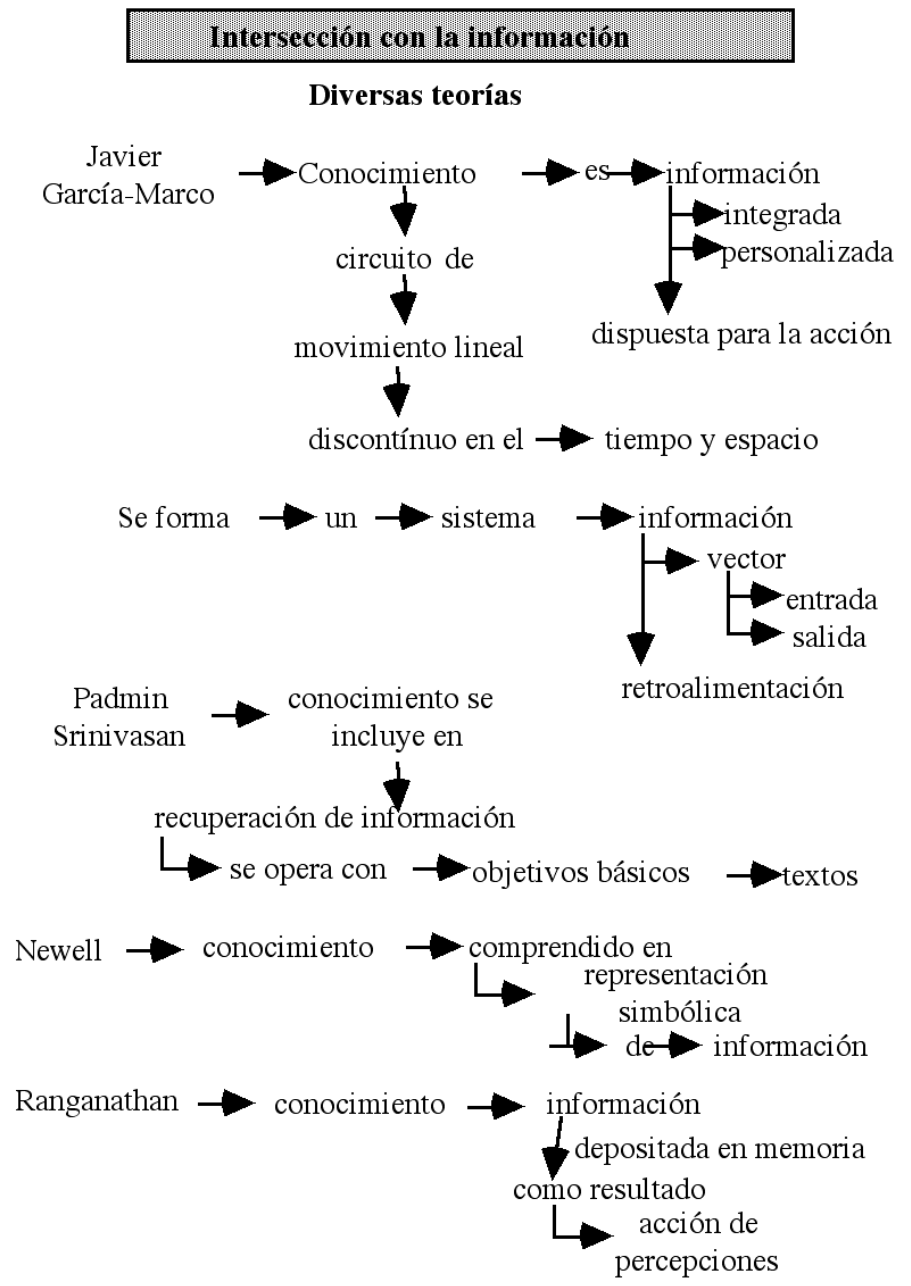

Figura 4

bles y transformables (Fig 5).

\section{La información}

De lo que se lleva escrito se puede deducir que aparecen diferentes opiniones

Scire. $1: 1$ (en.-jun. 1995). 
sobre la información. Puede ser interesante aclarar ciertos puntos antes de seguir adelante.

Todo el mundo habla de la información como algo propio, que nos pertenece y que usamos con la mayor naturalidad. Pocos se paran a pensar qué es, o puede ser; cuál es su naturaleza; sus propiedades... Ciertamente, la bibliografía que trata de la información en sí, es muy abundante, sobre todo con la vista puesta en su utilización y procesamiento. No abunda tanto la que se refiere a sus estudios teóricos.

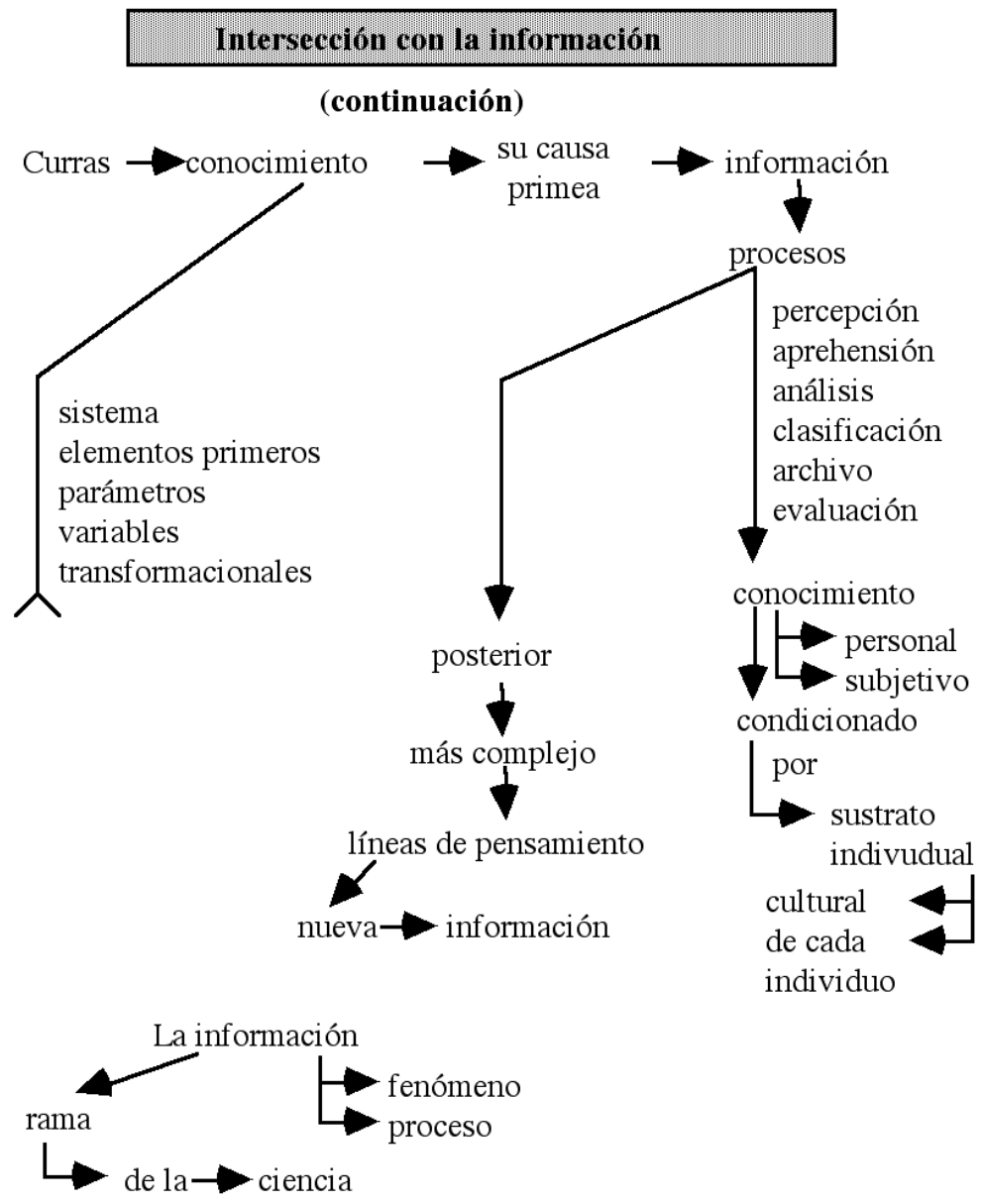

Figura 5

Scire. $1: 1$ (en.-jun. 1995). 
La información es todo y es nada al mismo tiempo. Existe, pero no se puede tocar. Es inherente a nuestra propia naturaleza humana. Sin ella no podríamos desarrollarnos; es energía que mueve las neuronas; es un bien de consumo... La información debe ser transmitida, comunicada y percibida; debe ser dominada, controlada y tratada para su adecuada utilización. En varios escritos he hecho referencia a la información considerada como fenómeno y como proceso. En el primer caso se trata de aquella que nos rodea, que nos impacta consciente o inconscientemente, independiente de nosotros mismos. Existe otra información querida y deseada que se elabora desde unos datos, contenidos allí donde de encuentren. Se confunde dato con información. Téngase en cuenta, que mientras el dato, no es transmitido, percibido y utilizado - no siempre pragmáticamente- no se convierte en información, en mensaje. El conjunto de procesos necesarios para transformar el dato en información, sumados al estudio de los documentos, de cualquier tipo y forma, que contienen aquéllos, incluyendo, conservación, análisis , clasificación, etc... constituye el cuerpo doctrinal y práctico de lo que entendemos por ciencias de la documentación. Se trata de una disciplina globalizadora, sistémica, en la que se incluyen la archivología, la bibliotecología y la documentación. Unidad de métodos, diferencias en los tipos de documentos y datos manejados.

Por otra parte, la información, con todo su bagaje anexo de sofisticadas tecnologías, métodos de trabajo para su tratamiento y dominio, lenguajes terminológicos con finalidad clasificatoria, etc, llegan a conformar una rama más del saber humano, donde se configura una doctrina teórica metódica y lógicamente formada, seguida de unas aplicaciones prácticas concretas y, en modo alguno, especulativas.

Estamos ante conceptos diferentes que reciben el mismo nombre. Aquí es donde se origina la confusión. La costumbre, la tradición empujan hacia el uso del vocablo "información" para todo. Se han hecho intentos de utilizar otras denominaciones, que chocan con aquellos muros y no prosperan. Puede ser muy recomendable tener todas estas ideas en cuenta, cuando se reflexiona sobre información, conocimiento y la organización de éste.

\section{Organización del conocimiento}

Ha resultado interesante constatar que en las enciclopedias más al uso, no figura el concepto "organización del conocimiento". Hay que dirigirse a los vocablos "ciencia", "saber" o el propio "conocimiento" para encontrar una referencia a su posible "organización". Por el contrario, acudiendo a publicaciones muy recientes, se encuentran abundantes referencias al tema. Es de suponer que esta aparente anomalía se deba, como se apunta anteriormente, a la gran difusión, de los últimos tiempos, del término inglés "knowledge" y su uso en informática y

Scire. 1 : 1 (en.-jun. 1995). 
cibernética. Asimismo se ha constatado que en el gran ámbito documentariobibliotecario, apenas si aparece otra referencia a la "organización del conocimiento" que no esté relacionada con la estricta clasificación de documentos y los temas a los que hacen referencia. En la mayoría de los casos "sistema de clasificación" — temática—, figura como sinónima la "organización del conocimiento". Efectivamente, el conocimiento conforma el área temática contenida en los documentos, de ahí la íntima concordancia entre "clasificación" y "organización".

Sin embargo, si se tienen en cuenta las diferentes acepciones del concepto de conocimiento, se ha de admitir que detrás de su "organización" existe un contenido más profundo, tanto filosófico, como práctico. En este escrito se hará referencia a las teorías más conocidas, a una somera evolución histórica, a su ámbito actual, a su propia clasificación y a sus formas de representación.

Para formular algunas reflexiones sobre contenidos y evolución en el tiempo, de la organización del conocimiento, se debe uno retrotraer a los estudios realizados sobre organización del saber, clasificación de las ciencias o teoría del conocimiento, e ir considerando las distintas ideas y postulados expuestos por los diferentes pensadores provenientes de las más variadas culturas y países (Fig 6).

\section{Su evolución en el tiempo}

Siguiendo las ideas de Rafael Rodríguez Delgado, en líneas generales, se pueden establecer varios períodos de evolución; partiendo de un primer período histórico, caracterizado por una forma de conocimiento global, donde, principalmente, arte, poesía, pintura, música, religión, filosofía, astronomía, geografía y agricultura se hallarían integradas, cuya representación más genuina son las cuevas de Altamira y de Stonehenge.

Seguiría un segundo período de diferenciación incipiente, que se inicia con los filósofos griegos y que llega hasta el siglo XIX, mostrando movimientos pendulares de la globalización general a una especialización un tanto acusada.

El tercer período se caracterizaría por una especialización progresiva tanto de los conocimientos teóricos, como de los prácticos. Su punto más prominente habría de situarse coincidiendo con aquél de la revolución industrial.

Como consecuencia se fijaría un cuarto período, en el que se acusa una gran especialización en el conocimiento. Surgen más y más disciplinas y se subdividen las existentes. Esta misma atomización empieza a provocar una nueva integración para no perderse en el desconcierto de tantos temas nuevos. Va surgiendo la idea de "ciencias interdisciplinares", pues no es fácil fijar compartimientos estancos. Distintos temas pueden ser estudiados desde distintos ángulos.

El quinto período, tan reciente, como antiguo, se caracteriza por la vuelta a la

Scire. 1 : 1 (en.-jun. 1995). 
globalización, a la síntesis del conocimiento; ahora conservando cada disciplina, rama del conocimiento, su individualidad. Reaparece el concepto de "transdisciplinariedad" de los saberes y la idea de las relaciones sistémicas.

Actualmente nos encontrariamos en un sexto período, donde predomina la tendencia hacia la síntesis, de forma que se habla de "systems thinking" — término acuñado, entre otros por el joven pensador inglés M. C. Jackson-y que refleja una "manera de pensar desde un punto de vista sistémico" o "pensamiento sistémico". Hoy en día, ninguna actividad se escapa a su enfoque sistémico.

A pesar de esta forma condicional de exposición, podemos tomar los períodos enunciados como reales. De hecho, eso ha sucedido así, con las variaciones o excepciones puntuales que hayan podido surgir en cada caso, debidas a pensadores aislados.

El mayor número de los períodos citados, la más rápida evolución, ha tenido lugar en los dos últimos siglos; y aún se seguirán grandes cambios en los próximos tiempos entrando en el siglo XXI.

Si se tienen en cuenta los estudios sobre epistemología (teoría del conocimiento), ya utilizados por Aristóteles, se pueden concretar diferentes tendencias. Cada una de ellas ha centrado la organización del conocimiento en un postulado diferente. Así han ido surgiendo el racionalismo, el empirismo, el determinismo, el positivismo, el naturalismo, el existencialismo... hasta llegar al informacionismo actual. También se habrá de considerar la epistemología científica, cuyo fundador en España fue Angel Amor Rubial o la metafísica del conocimiento nacida modernamente hacia finales del siglo XIX, o la gnoseología, ésta expuesta por Alexandre Gottfiel Baumgarten, ya en el siglo XVIII. En España, en el siglo XIX, introdujo la gnoseología F. Ferrier, como una teoría del conocimiento del saber científico.

La última, novísima, línea de pensamiento que ha aparecido es la contenida en la "filosofía sistémica" que sitúa la organización del conocimiento en el centro del ámbito de la ciencia de sistemas, en concordancia con el pensamiento sistémico, unificador e individualizador a la vez. Esto supone colocar aquél, en un plano de abstracción mayor, en un nivel superior en las líneas de pensamiento (Fig 6).

\section{Su formación}

El paso siguiente para llegar a comprender lo que supone la organización del conocimiento, será estudiar la manera en que llega a formarse, tanto para constituir el propio acerbo cognoscitivo, como el acerbo cultural colectivo.

Sintetizando ideas de varios pensadores como por ejemplo Wordsworth o P. S. K. Sharma, se podría fijar la organización del conocimiento individual en los Scire. 1 : 1 (en.-jun. 1995). 
procesos mentales que comienzan con la percepción y continúan con el razonamiento, sentido común, intuición, asimilación y crítica, para terminar con la transmisión. En mi opinión, en cada una de esas etapas se va configurando una organización parcial y peculiar del conocimiento, que no llega a su plenitud hasta que no es transmitida. Esta última fase puede ser discutida, toda vez que los seres humanos no siempre exteriorizan sus pensamientos.

Aquellos procesos se corresponden con otros tantos niveles de actividad

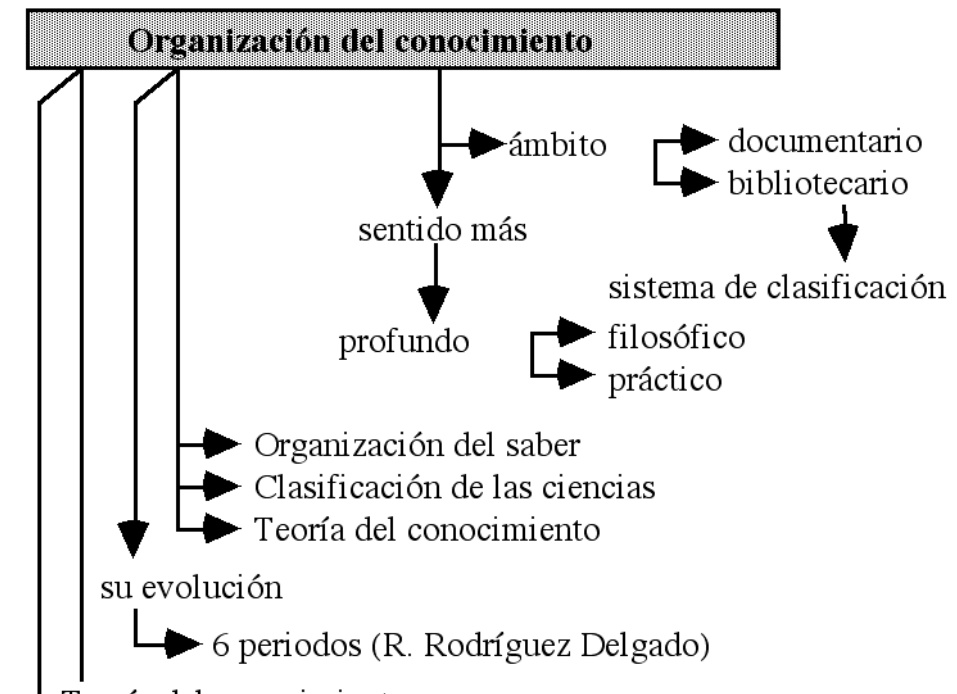

Teoría del conocimiento

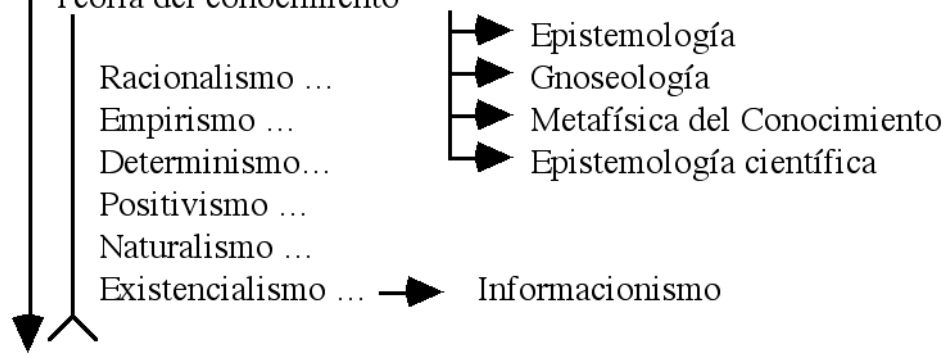

Pensamiento sistémico $\rightarrow$ Filosofía sistémica

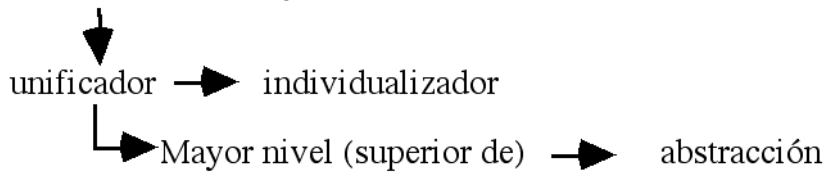

Figura 6

Scire. $1: 1$ (en.-jun. 1995). 
mental, posterior, más compleja, determinada por las fases de conocimiento, comprensión, interpretación, extrapolación, aplicación, análisis, síntesis y evaluación fijadas entre otros por Mamota Das.

Teniendo en cuenta los procesos y los niveles mentales citados se pueden establecer ciertas formas de organización del conocimiento, por otra parte ya conocidas desde antiguo; pero que conviene traer de nuevo a la memoria para la mejor exposición de los razonamientos de este escrito. Así, se establece aquella formando los binomios:

- sensible

- inteligente

- intuitiva

- racional

- inmediata

- mediata

- a priori

- a posteriori

- intelectual

- emotiva

Estos grupos se pueden reunir en otros dos de mayor ámbito:

- especulativo

- pragmático

Con todo ello se alcanza una visión de conjunto referente a la formación de la organización de conocimiento (Fig 7).

\section{Su ordenacion}

Desde el punto de vista de nuestra cultura occidental, la tendencia general para establecer una ordenación de la organización del conocimiento, ha sido distribuir éste en dos grandes clases pertenecientes al mundo inteligente y al mundo sensible. El mismo Platón en su dualismo metafísico, fija esa distinción. Aristóteles añade un tercer elemento práctico en su esquema... teórico, práctico y poético (productivo). Estas líneas con más o menos variaciones, subdivisiones y añadidos ha prevalecido hasta nuestros tiempos. Si bien, las recientes, posteriores teorías se inclinan por una organización del conocimiento distribuida entre pura, aplicada y del espíritu.

La más rabiante actualidad se decanta por la unidad del pensamiento, donde caben tantas subdivisiones, ramas del saber, o disciplinas, como están surgiendo, 
fruto del gran desarrollo científico y técnico actual.

NOTA: Se apreciará, que en la exposición de estos razonamientos se aplican las teorías e ideas utilizadas por filósofos y científicos para clasificar las ciencias; y es que, en este caso, se entiende "conocimiento" en su segunda acepción, como acumulación de saber. Por tanto, puede ser comparado al concepto de ciencia, aunque con aquella variante de subjetividad que se apunta al principio.

\section{Organización del Conocimiento}

su formación
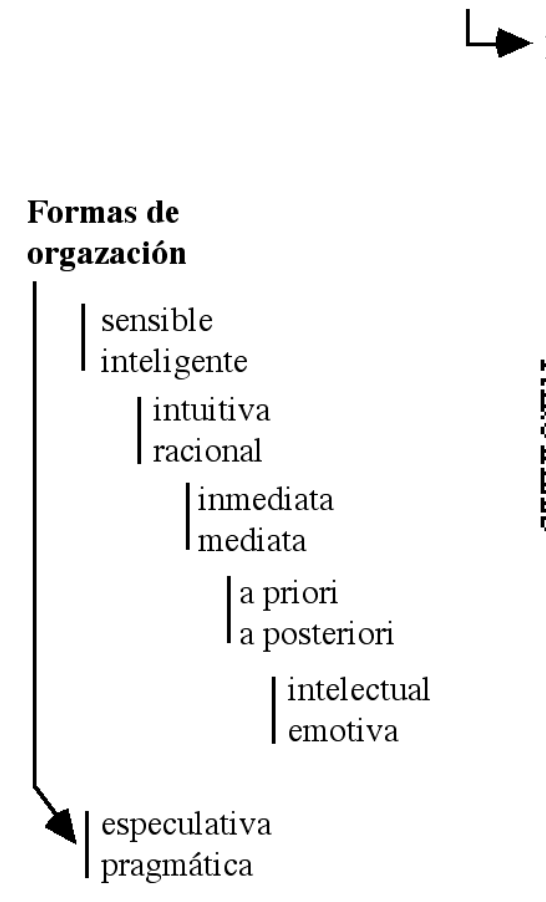

para construir acervo

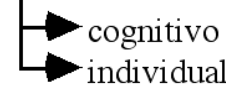

Figura 7

Scire. $1: 1$ (en.-jun. 1995). 


\section{Su representación}

El estudio de la presentación y representación de la organización de conocimiento, se debe contemplar bajo el prisma de la propia presentación y representación del conocimiento. En realidad, supone ser un apartado importante dentro de la epistemología, o de la gnoseología, que se ha tratado desde antiguo, aunque hayan sido ampliados su ámbito y sus aplicaciones.

Teniendo en cuenta el carácter subjetivo del conocimiento y su formación primera en la mente del hombre, los filósofos y psicólogos han centrado sus estudios en las representaciones mentales internas de los individuos, elucubrando sobre cuales pueden ser las representaciones conscientes o inconscientes, que influyen en el comportamiento; y que como tales elucubraciones se prestan a variadas interpretaciones y construcción de modelos. Asimismo, se tienen en cuenta representaciones de tipo biológico y social, por cuanto que las personas viven en estado asociado.

Lo que aquí interesa ahora es recordar esos tipos de representaciones, sin profundizar en detalles, ya que el propósito de este escrito es realizar una exposición de la situación general.

Hacia el exterior, los seres humanos comunican sus representaciones de su conocimiento por vía oral, escrita o gráfica, que se traduce en la utilización de lenguajes asimismo orales, escritos y gráficos. Fijando la atención, de momento, en los segundos, se distingue entre lenguajes naturales y artificiales.

Actualmente, la gama de lenguajes artificiales que se han creado y se crean cada día, crece constantemente. Es un campo que se encuentra en pleno surgimiento y evolución, principalmente por la explosión de la información y la aplicación de la computación. Tanto es así, que se ha hecho necesario recapitular sobre el asunto y establecer una cierta organización. García Gutiérrez dirige sus investigaciones hacia estos temas.

En mi obra sobre tesauros, se dedican unos pocos capítulos al tema de la terminología y allí aparecen variadas definiciones de lenguaje. Si se toma aquella que hace referencia a una "representación simbólica del conocimiento" se puede establecer el esquema siguiente:

- representación simbólica del conocimiento

- lenguajes

- natural

- artificial

- codificado (p.e. programas informáticos)

- terminológico

Scire. 1 : 1 (en.-jun. 1995). 
- vocabulario especial

- documentario

- sistema clasificatorio (p.e. CDU, tesauros)

Si se admite que un sistema de clasificación del contenido temático de un documento, es un lenguaje codificado, se habría de escribir el esquema de otra forma. Sin embargo, lo que interesa, ahora, es poner de manifiesto que un lenguaje informático también sirve para representar el conocimiento y que representa una forma de su organizar.

Se enlaza, de esta manera, la organización del conocimiento con la informática, la cibernética y las nuevas tecnologías aplicadas al tratamiento de la información.

Para redondear este apartado, cabe aún mencionar ciertas formas de representación mental, que tiene su correspondencia en una presentación gráfica, como por ejemplo, las más habituales que se citan a continuación:

- dicotómica

- policotómica

- arborescente

- jerárquica

- asociativa

Las representaciones gráficas, como tales, pueden ser tan variadas, como variada es la imaginación del ser humano. Cada uno utiliza aquella que mejor sirve a sus propósitos, aunque las más usadas suelen ser las cuadrangulares, las circulares y las espirales. En cualquier caso, no existen normas establecidas al efecto.

\section{Ambito actual de la organización del conocimiento}

He aquí el concierto y el desconcierto en la organización del conocimiento actual. Por un lado prevalecen las teorías filosóficas y psicológicas práctico-especulativas y por otro lado surgen las nuevas ideas consecuencia de las aplicaciones informáticas a las ciencias de la documentación, tomadas éstas en su sentido más amplio.

Quizá haya que atribuir esa dicotomía, discordante y concordante al mismo tiempo, al desplome de los grandes principios tenidos como fundamentales durante siglos. Hoy en día predomina la relatividad, la indeterminación, se admite una nueva concepción espacio-temporal; se modifica la noción de las partículas elementales, de la naturaleza de la energía; han cambiado las concepciones matemáticas... En general predomina una gran flexibilidad en lo que se refiere a 
la concepción del universo y sus principios fundamentales. Se está predispuesto al cambio y a la modificación de las ideas. Por añadidura, los adelantos científicos y tecnológicos traen nuevos campos de pensamiento, nuevas especialidades y nuevas aplicaciones, con los correspondientes nuevos utensilios. Para poder hacer frente a estas situaciones se recurre a contrastar lo viejo con lo nuevo y aprovechar aquello para mejorar el rendimiento de ésto.

Lo referente a la organización del conocimiento, como causa primera y fin último de las actividades humanas, no se libra de los influjos en vigor. Su campo de acción se ha ampliado. En primer lugar, las corrientes modernas contemplan la organización del conocimiento desde dos grandes ámbitos: uno como tal y otro bajo el signo de su utilización. Aquél se conserva en sus principios filosóficospsicológicos con pocas modificaciones. El segundo aspecto se manifiesta muy afectado. Por de pronto, hoy se habla de la organización del conocimiento industrial, empresarial, social, científica, documentaria, bibliotecaria, informática... es decir, aplicada a las tareas diarias más corrientes e imprescindibles, incluso John P. Van Gigch habla de "ciencias de la decisión".

Como consecuencia de esa realidad, estudiosos e investigadores han dado en profundizar en el tema, con la subyacente idea globalizadora actual y han establecido un nuevo campo del saber, denominado "ciencias cognitivas". Los antiguos paradigmas no se adecuan a las circunstancias modernas y han elaborado un nuevo "paradigma cognitivo", al amparo del cual, Ingetraut Dahlberg sitúa sus "unidades cognitivas" y establece los "campos cognitivos", donde se sitúan las "estructuras cognitivas" (Fig 8).

Si nos paramos a pensar un poco, nos daríamos cuenta de que se trata de los mismos conceptos vistos desde el ángulo de la computación y las tecnologías informáticas, utilizando una nueva terminología.

Los científicos de la información, los documentalistas y los bibliotecarios toman esas nuevas ideas para sí y las definen en función de sus principios y necesidades. Por ejemplo, John P. Van Gigch asocia las ciencias cognitivas a la representación de símbolos en informática. Neelemeghan dice que cuando se produce un desfase entre un conocimiento tenido como cierto y un nuevo conocimiento, que también aparece como cierto, se debe buscar un paradigma cognitivo que relacione ambos... y que permita su tratamiento simbólico. Para I. Dahlberg las "unidades cognitivas" equivalen a los conceptos, expresados por una o varias palabras; las "estructuras cognitivas" se equiparan a los encabezamientos de los sistemas clasificatorios y los "campos cognitivos" a las diferentes clases de estos sistemas. Por su parte, Gopinath habla de los nuevos métodos de búsqueda de información, más asequibles al usuario, que requieren nuevos "modelos cognitivos" de búsqueda. Utilizando la terminología clásica, se hablaría de "estrategias

Scire. $1: 1$ (en.-jun. 1995). 
de búsqueda". En ese sentido R. Fugmann define el "paradigma cognitivo" en la organización del conocimiento como una estructura del conocimiento y sus componentes, que presente flexibilidad en el entendimiento y asimilación de la demanda de información. En agosto de 1992 se ha celebrado un congreso en Madrás sobre "los nuevos paradigmas en la organización del conocimiento" donde la mayoría de los trabajos trataban de la estructuración de muy diversos sistemas clasificatorios.

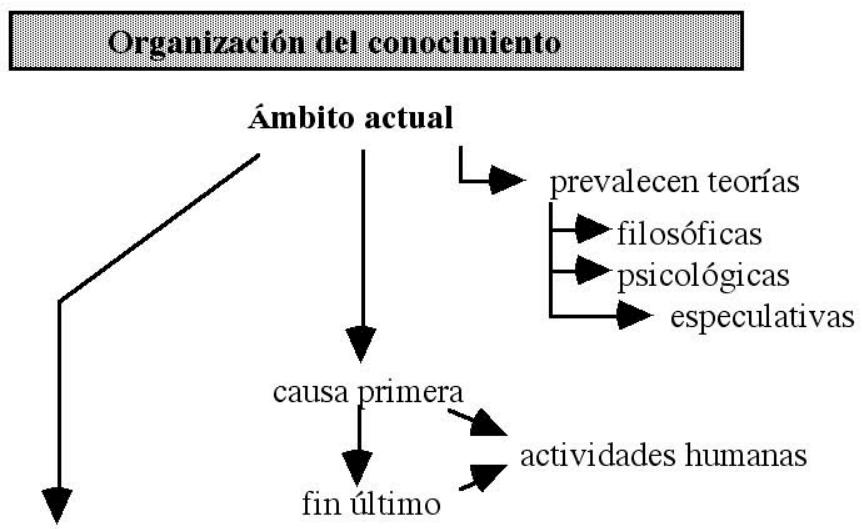

grandes cambios

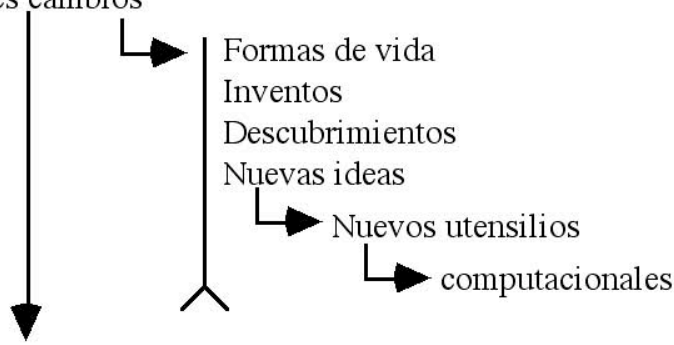

Organización del conocimiento

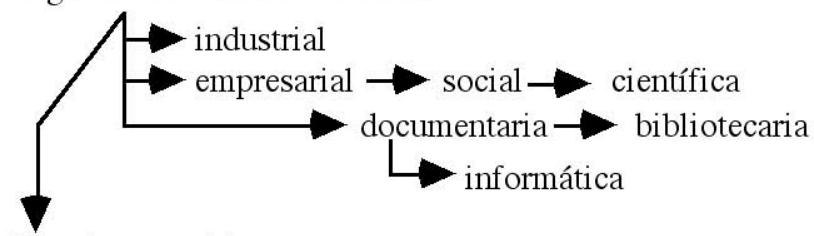

Ciencias cognitivas

idea globalizadora

Figura 8

Scire. $1: 1$ (en.-jun. 1995). 
Aquí y en términos generales se postula que las ciencias cognitivas, como ciencias interdisciplinares, deben basarse en la psicología, la gnoseología, los estudios de comportamiento, la informática, la inteligencia artificial, y las ciencias documentarias. Todo ello con un criterio amplio y abierto a nuevas corrientes de opinión y a la controversia y la crítica. La organización del conocimiento deberá estar en consonancia con las necesidades de la sociedad en cada momento (Fig 9).

\section{Tendencias hacia el futuro}

La irrupción de las ciencias de la computación — nueva rama sistémica del saber, con su nueva terminología - en el campo de la organización del conocimiento está provocando la aparición de, asimismo, otras nuevas ramas del saber que se van concretando, poco a poco, en sus principios teóricos y en sus aplicaciones. La ingeniería del conocimiento, la bio-informática, la bio-computación, la eco-informática, la agro-informática, etc, son algunas de estas nuevas ciencias, que se van perfilando hacia el futuro.

NOTA: Conviene hacer, en este punto, una precisión con respecto a la "bioinformática", que está empezando a utilizarse, muy recientemente, como traducción de la expresión inglesa "informational biology". No es fácil encontrar un equivalente en el castellano utilizado en España, del vocablo "informational", que de ningún modo significa información; más bien se puede interpretar como "información informatizada". Aunque tampoco es correcto ya que la informática no abarca todas las aplicaciones de los circuitos electrónicos, ni las llamadas nuevas tecnologías, con la inteligencia artificial al frente. Es más adecuado y completo el vocablo "computación" que se usa en los países americanos de habla hispana.

Una vez hecho este inciso, volvamos con nuestra organización del conocimiento, que en estas nuevas concepciones y aplicaciones se muestra muy adecuada para serle aplicados los principios de la ciencia de sistemas. Por este lado es por donde se vislumbra realmente el camino de los estudios teóricos y las experimentaciones. En la Universidad de Zaragoza, los jóvenes investigadores Javier García, Miguel Angel Esteban y Antonio Paulo Ubieto han comenzado ya esas líneas de investigación, lo mismo que algunos compañeros de la Universidad de Murcia. Por mi parte me dedico desde hace unos pocos años al estudio de la ciencia sistémica y sus aplicaciones a las ciencias documentarias, exponentes de la organización del conocimiento (Fig 9).

\section{Proyeccion docente}

A lo largo de todo este escrito se ha venido hablando de la organización del

Scire. $1: 1$ (en.-jun. 1995). 
conocimiento, desde diferentes ángulos. También se ha puesto de manifiesto la importancia que ha adquirido en los últimos tiempos, debido principalmente a su inserción en las ciencias de la computación, como elemento primero, base de conocimiento; pero asimismo como elemento imprescindible en la conservación y utilización del acerbo científico y cultural de los pueblos, por medio de la información.

A tenor de esos cambios y modificaciones y aplicaciones ha surgido un nue-

\section{Organización del conocimiento}

Su ámbito actual (continuación)

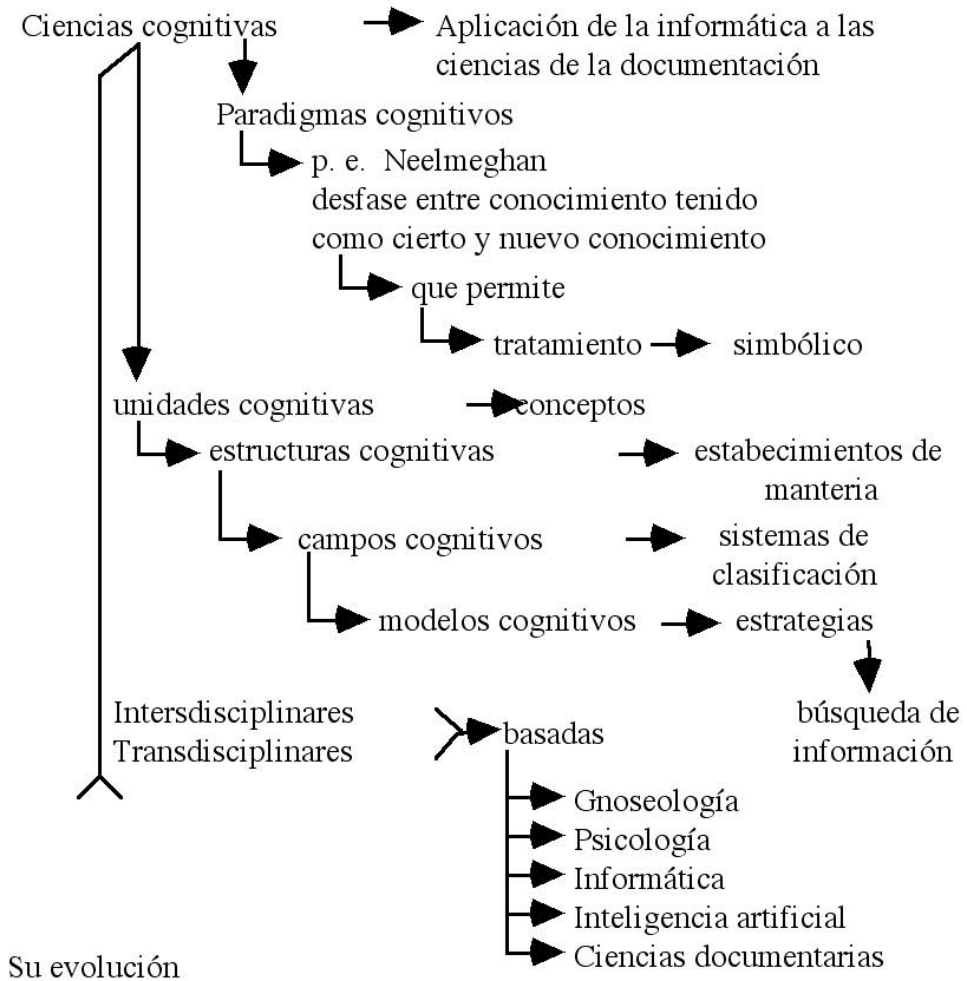

Su evolución

necesidades de su tiempo

Figura 9

Scire. $1: 1$ (en.-jun. 1995). 
vo "paradigma cognitivo", que fundamenta un nuevo conjunto de "ciencias cognitivas". Se vislumbra un moderno "panorama cognitivo" estudiado desde niveles de abstracción más elevados, entre los que se cuentan los postulados sistémicos.

Si realmente esas son las tendencias actuales y futuras, parece adecuado, primordial, que se incluyan en las enseñanzas universitarias. A ese respecto, se deben mencionar las iniciativas de los jóvenes investigadores, ya citados, del

\section{Organización del conocimiento}

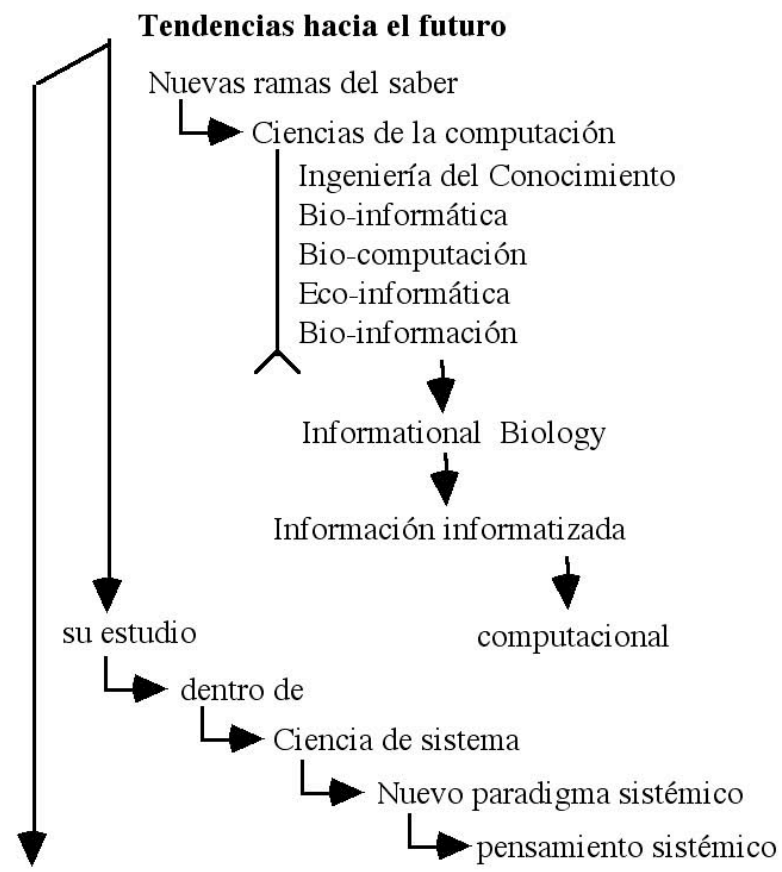

Proyecto docente

"Fundamentos de representación, comunicación y organización del conocimiento"

"A la organización del conocimiento por la Organización del Conocimiento"

Figura 10

Scire. $1: 1$ (en.-jun. 1995). 
área de Biblioteconomía y Documentación de la Universidad de Zaragoza, quiénes tienen el proyecto de introducir una nueva asignatura en sus planes de estudio, titulada "Fundamentos de representación, comunicación y organización del conocimiento" (Fig 10).

Iniciativas, como esta, que pretenden poner en contacto los estudiantes universitarios con pensadores de diversas disciplinas, deberían ser fomentadas y auspiciadas, igualmente en otras Universidades españolas. Estarían en la vanguardia de las corrientes de pensamiento internacionales, marcando pautas a seguir para investigadores futuros. Parece que se pueden adoptar posturas optimistas y se caminará por esos derroteros.

\section{Conclusión}

Después de todo lo aquí escrito, da la impresión de que sólo queda lugar para esbozar una somera síntesis de conjunto. Se ha tratado la organización del conocimiento partiendo del propio conocimiento. Se ha estudiado aquella, desde puntos de vista filosóficos, tenidos como clásicos. Se ha realizado un recorrido por las diferentes teorías sobre la organización del conocimiento, la formación de ésta, su ordenación y su representación.

Se ha puesto de manifiesto la dualidad actual, pues se sigue estudiando la organización del conocimiento desde su perspectiva filosófico-psicológica, al mismo tiempo que ha surgido un nuevo campo, denominado ciencias cognitivas. Estas son principalmente fruto de las aplicaciones informáticas a las ciencias documentarias. Se vislumbran, igualmente otras aplicaciones.

En definitiva, se ha ampliado el ámbito teórico y práctico de la organización de conocimiento, que se contempla desde un nivel mayor de abstracción globalizador y particularizante al mismo tiempo, donde caben los estudios en su enfoque sistémico.

Se pretende, con este escrito, ordenar ideas y traer un poco de claridad a un campo del saber en plena evolución y en plena turbulencia. A la organización del conocimiento por la organización del conocimiento.

Madrid, Septiembre de 1992

\section{Referencias}

A) Consultadas

Abbagnano, N. Diccionario de Filosofía.. México ; Buenos Aires : Fondo de Cultura Económica.

Curras, E. Information Science- Information as a Dialectic and Interactive System. // Cognitive Paradigms in Knowledge Organization. Madrás : Sarada R.E. for Library

Scire. 1 : 1 (en.-jun. 1995). 
Science, 1992. 418-431.

Curras, E. The Vertical Integration of Science as a Stabilizing Factor for Mankind. // Intern. Systems Science Handbook. / by R. Rodríguez and B.H. Samos : Banathy. University of the Aegean, 1992. 3.7-3 7.17.

Curras, E. Tesauros: Importantes Herramientas de trabajo. Su relación con los lenguajes terminológicos. // Tecnolegis. 10 (en.-marzo 1992) 3-11.

Curras, E. Thesauros, Lenguajes Terminológicos. Madrid : Paraninfo, 1991.

Curras, E. Un Nuevo Concepto de Información en la Integración Científica. // Actas 45th Conference and Congress FID. La Habana. Septiembre 1990. 10-13.

Curras, E. El Dato Informativo en la Industria Alimentaria. // Actas I Congreso Internacional de Tecnología y Desarrollo de Alimentos. 1990. 10-14.

Curras, E. Dialectic Interaction in Science. // Actes du Congrès Europèen de Systemiquè. Association Française pour la Cybernetique Economique et Technique. Lausanne. Oct 1989, e INICAE ,9,1. Marzo 1990. 5-17.

Curras, E. La Información en sus Nuevos Aspectos. Ciencias de la Documentación. Madrid : Paraninfo, 1988.

Curras, E. Information as the Fouth Vital Element and its Influence on the Culture of People. // J.Inf. Science. 13 : 3 (1987), 149-157.

Curras, E. Las Ciencias de la Documentación : Bibliotecología, Archivología, Documentación, Información. Barcelona : Mitre, 1988.

Dahlberg, I. The Network of Knowledge Fields: Conceptual Systematization in Action. // Cognitive Paradigms in Knowledge Organisation. Madras : Sarada R.E. for Library Science, 1992. 323-333.

Das, M. Learning Teaching Cognitive Paradigms in Knowledge Organization. // Cognitive Paradigms in Knowledge Organisation. Madras : Sarada R.E. for Library Science, 1992. 362-371.

Elzaburu, F.; Martitegui, J. La crisis Mundial. De la Incertidumbre a la Esperanza. Espasa Calpe : Madrid, 1988.

Enciclopedia Filosófica. Istituto per la collaborazione Culturale. Venezia, Roma.

Ferrater Mora, J. Diccionario de Filosofía. Buenos Aires, 1973.

Fugmann, R. Tools for Knowledge Organization and the Human Interface. I ISKOConference. Darmstadt. Aug. 1990. 14-17.

García Gutiérrez, A. Teoría del Lenguaje Documental. // Estructura lingüística de la documentación, Teoría y Método. Murcia: Universidad, 1990. Cap. 3.

García Marco. F.J. Principios del Análisis y Representación del Contenido. // Teoría General de la Documentación. Zaragoza : Universidad, 1992.

García Redondo, L. Definiciones del Concepto de Información en España: 1970-90. Madrid : Universidad, Curso de Documentación Pedagógica, Mayo 1992.

Gran Enciclopedia RIALP XIX. Madrid : Rialp, 1974.

Marijuán, P.C.; Westley, J. Enzymes as Molecular Automata: A reflection on some numerical and philosophical aspects of the hypothesis. // BioSystems. 26 (1992). En prensa.

Scire. 1 : 1 (en.-jun. 1995). 
Marijuán, P.C. Enzymes and Theoretical Biology: Sketch of an informational perspective of the cell. // BioSystems. 25 (1991) 259-273.

Mayor Zaragoza, F. Ciencia y Desarrollo Humano. // AFINAE 49 : 439 (1992) 159-164.

Narasimhan, R. Cognitive Paradigms in Knowledge Organization : 2nd ISKO Conference : Madras, Agosto 1992. Madras : Sarada R.E. for Library Science, 1992.

Newell, A. The Knowledge Level. // Artificial Intelligence. 18 (1928) 87-127.

Parameswaran, M. Propaedia of Encyclopaedia Britannica: A Conceptual Model of knowledge Organisation. // Cognitive Paradigms in Knowledge Organisation. Madras : Sarada R.E. for Library Science, 1992. 260-268.

Petrovski,A. Age group and Pedagogical Psychology. Moscow : Progress Publishers, 1986.

Ranganathan, S.R. Prolegomena to Library Classification by S.R. Ranganathan. .Assis by M.A. Gopinath. Bombay : Asia Publishing House, 1967. 81.

Rodrígueza Delgado, R.; Bela, H.B. International Systemas Science Handbook. Samos : University of Aegean, 1992.

Seetharama, S. Cognitive Approach in Information Consolidation. // Cognitive Paradigms in Knowledge Organisation. Madras : Sarada R.E. for Library Science, 1992. 286-292.

Sharma. S.K. Universe of Knowledge and Research Methodology. Delhi : Ken Publications, 1990.

Shrivastava, R.K. Knowledge Generating Process: A Conceptual Model in Ecosystematic. Framework. // Cognitive Paradigms in Knowledge Organisation. Madras : Sarada R.E. for Library Science, 1992. 432-44

Srinivasan, P. Knowledge Organisation for Information Retrieval. // Cognitive Paradigms in Knowledge Organisation. Madras : Sarada R.E. for Library Science, 1992. 81-94.

Svenonius, E. The Impact of Computer Technology on Knowledge Representation. // Cognitive Paradigms in Knowledge Organisation. Madras : Sarada R.E. for Library Science, 1992. 1-15.

Szent Gyorgyi, A. Bioelectronics. New York : Academic Press, 1968.

B) Encontradas

Bliss, H.E. Organization of Knowledge and the System of the Sciences. New York : H.W. Wilson \& Co., 1929.

Gardner, H. The Mind's New Science: A History of the Cognitive Revolution New York : Basic Books, 1985.

Godert, W.; Jaencke, P. Kognitive Anstze zum Ordnen und Darstellen von Wissen. // 2 Tagung der Deutschen ISKO Secktion. Weilburg, oct. 1991. 15-18.

Minsky, M. A Framework for Representation Knowledge. : The Psychology of Computer Vision, by P. Winston. New York : Mc Graw Hill, 1975.

Shera, J.H. Libraries and the Organisation of Knowledge. Hamden : Archon Books, 1956. Winograd, T. ; Florres, F. Understanding Computers and Cognition. Ablex, 1986.

Scire. 1 : 1 (en.-jun. 1995). 